\title{
Computational Molecular Docking and Virtual Screening Revealed Promising SARS-CoV-2
}

Drugs

Maryam Hosseini ${ }^{1}$, Wanqiu Chen ${ }^{1}$, Charles Wang ${ }^{1,2^{*}}$

${ }^{1}$ Center for Genomics, School of Medicine, Loma Linda University, Loma Linda, CA 92350, USA ${ }^{2}$ Division of Microbiology \& Molecular Genetics, Department of Basic Sciences, School of Medicine, Loma Linda University, Loma Linda, CA 92350, USA

*All correspondence should be addressed to: CW at chwang@llu.edu or oxwang@gmail.com

\section{Abstract}

The pandemic of novel coronavirus disease 2019 (COVID-19) is rampaging the world with more than 1.4 million of confirmed cases and more than 85,000 of deaths across world by April $9^{\text {th }}, 2020$. There is an urgent need to identify effective drugs to fight against the virus. Severe acute respiratory syndrome coronavirus 2 (SARS-CoV-2) belongs to the family of coronaviruses consisting of four structural and 16 non-structured proteins. Three non-structural proteins such as main protease, papain like protease, and RNA-dependent RNA polymerase are believed to play a crucial role in the virus replication. We applied a computational ligand-receptor binding modeling and performed a comprehensive virtual screening on the FDA-approved drugs against these three SARS-CoV-2 proteins using AutoDock Vina. Our computational studies indicated that Simeprevir, Ledipasvir, Idarubicin, Saquinavir, Ledipasivir, Partitaprevir, Glecaprevir, and Velpatasvir are all promising inhibitors, which displayed a lower binding energy (higher inhibitory effect) than Remdesivir, Lopinavir, and Ritonavir. However, we found that chloroquine and hydroxychloroquine, which showed efficacy in treating the COVID-19 in recent clinical studies, had high binding energy with all three proteins, suggesting they may work through a different mechanism. We also identified several novel drugs as potential inhibitors against SARS-CoV-2, including antiviral Raltegravir; antidiabetic Amaryl; antibiotics Retapamulin, Rifimixin, and Rifabutin; antiemetic Fosaprepitant and Netupitant. In summary, our computational molecular docking approach and virtual screening identified some promising candidate SARS-CoV-2 drugs that may be considered for further clinical studies.

\section{Key Points:}

- Computational molecular docking identified potential promising SARS-CoV-2 drugs. 
- Three SARS-CoV-2 non-structural proteins Mpro, PLpro, and RdRp were simultaneously targeted to study the protein-inhibitor binding energies.

- Raltegravir, Amaryl, Retapamulin, Rifaximin, Rifabutin, Fosaprepitant, and Netupitant were found to have low binding energies with three SARS-CoV-2 non-structural proteins.

- Remdesivir, Lopinavir, and Ritonavir were also found to have low binding energies with SARSCoV-2 non-structural proteins, while chloroquine and hydroxychloroquine had high binding energies with SARS-CoV-2 non-structural proteins suggesting that chloroquine and hydroxychloroquine may work through a different mechanism.

Keywords: COVID-19, SARS-CoV-2, protease, virtual screening, molecular docking

Abbreviations: nonstructural proteins: NSP; main protease: Mpro; papain-Like proteases: PLpro; RNA-dependent RNA polymerase: RdRp

\section{Introduction}

A novel coronavirus disease (COVID-19), first started in December 2019 in Wuhan, China ${ }^{1,2}$, has spread to more than 200 countries across world and the ongoing pandemic has become a global health emergency. As of April $9^{\text {th }}, 2020$, there have been more than 1.4 million of confirmed cases and 85,522 deaths globally (data from World Health Organization website), with a continuous on-going growth rate of $8 \%$. Similar to the SARS (Severe Acute Respiratory Syndrome) coronavirus and MERS (Middle East Respiratory Syndrome) coronavirus, the SARS-CoV-2 (Severe Acute Respiratory Syndrome 2) also belongs to the betacoronavirus (beta-CoV) family, RNA viruses with crown-like spikes on their surface of the coronavirus particles. The fatality rate of the new SARS-CoV-2 seems lower than that of SARS and MERS. An estimate of the overall fatality rate in China is $\sim 1.38 \%$ for SARS-CoV- $2^{3}$, whereas the World Health Organization (WHO) estimated the fatality rate of $\sim 14-15 \%$ for SARS ${ }^{4}$ and $\sim 35 \%$ for MERS 5 . However, SARS-CoV-2 has the features of rapid transmission from person-to-person, asymptomatic transmission ${ }^{6}$ and prolonged symptomatic development, as well as substantial increased fatalities in the aged group ${ }^{7}$. The pandemic of COVID-19 has caused a surged need for intensive care, which is giving rise to tremendous pressure on the healthcare systems in many countries.

Up to now, there is no FDA-approved or proven effective treatment for SARS-CoV-2 infection. The anti-coronaviral strategies include preventing the synthesis of viral RNA, inhibiting virus replication, blocking the virus binding to human cell receptors, or inhibiting the virus's self-assembly process ${ }^{8}$. SARS-CoV-2 genome encodes for four structural and 16 non-structural proteins (NSP) ${ }^{9}$. Among these translated NSPs, the main protease (Mpro), also called as the chymotrypsin-like protease (3C-like 
protease), and the papain-Like protease (PLpro) are the two essential proteases for proteolytic processing of the coronavirus replicase polyprotein therefore generating functional replication complex of the virus ${ }^{10,11}$; whereas RNA-dependent RNA polymerase (RdRp) is the central enzyme for RNAsynthesizing in all positive-strand RNA virus replication ${ }^{12}$. These three NSP proteins play crucial roles in coronavirus replication, making them attractive targets for anti-coronaviral drug design.

Substantial efforts have been made for treating SARS-CoV-2 infected patients ${ }^{13-15}$. Although dozens of potential therapies for SARS-CoV-2 have been suggested during the COVID-19 outbreaks, no therapeutics with proven clinical efficacy and safety has been established for treatment of COVID19. WHO have now launched a giant trial, SOLIDARITY, to focus on testing the four most promising COVID-19 treatments - Remdesivir; chloroquine and hydroxy-chloroquine; Lopinavir plus Ritonavir; and Lopinavir plus Ritonavir and interferon-beta. It is worth mentioning that the four therapies against SARS-CoV-2 are somewhat targeting one of the three NSPs proteins of coronavirus - Mpro, RdRp, and PLpro. Remdesivir is an approved human immunodeficiency virus (HIV) reverse transcriptase inhibitor, which has broad-spectrum activities against the RdRp of coronavirus, including MERS-CoV and SARS-CoV ${ }^{16}$. Lopinavir and Ritonavir, which act against the viral main protease (Mpro), have been shown to be effective in treating patients with SARS ${ }^{17}$ and MERS-CoV ${ }^{18,19}$. A randomized clinical trial of Lopinavir-Ritonavir efficacy in patients with COVID-19 has been carried in Wuhan, China ${ }^{20}$. The chloroquine and hydroxychloroquine, although used as antimalarial drugs for decades, have been reported as a potentially effective agent in the treatment of COVID-19 ${ }^{16,21}$. In addition to its well-known function of elevating endosomal $\mathrm{pH}$ to block virus and cell fusion, chloroquine also showed antiviral function at post-entry stage, which might be related to inhibiting coronavirus papain-like protease ${ }^{22}$. Overall, targeting one or multiple NSP proteins including Mpro, RdRp, and PLpro, will lead to potential treatment for COVID-19.

Computational screening of FDA-approved drugs with the potential of targeting SARS-CoV-2 is a cost-effective and less time-consuming strategy and can quickly identify promising ready-to-use candidates. Recently, molecular docking-and virtual screening methods have been attempted to identify potential drugs for COVID-19 by protein-ligand binding energy prediction. Due to the limitation in crystal structures for SARS-CoV-2 proteins, many studies have used the homology models generated based on SARS-CoV-2 genome and SARS crystal structure ${ }^{23}$ 24. Previous studies have screened the small molecules that target either of SARS-CoV-2 Mpro, PLpro, or RdRp proteins. Some potential candidates for SARS-CoV-2 have been identified, many of which are anti-HIV or hepatitis $\mathrm{C}$ (HCV) drugs. In this study, we aim to screen FDA-approved drugs that can inhibit one or more of the three SARS-CoV-2 Mpro, RdRp, and PLpro proteins, and attempt to identify other drug candidates with high binding affinities than Remdesivir or Lopinavir, or potential of binding to target multiple key 
functional proteins of SARS-CoV-2. In this regard, we conducted virtual ligand screening of 1615 FDAapproved drugs on the binding pocket of SARS-CoV-2 Mpro, PLpro, and RdRp proteins.

\section{Results}

\section{D structures of Mpro, PLpro, and RdRp for virtual screening}

In order to achieve the mode of interaction of FDA-approved drugs with the binding pocket of three different SARS-CoV-2 NSPs, we prepared the 3D structures of the Mpro, PLpro, and RdRp proteins. We fetched the newly released crystal structure of the SARS-CoV-2 Mpro (PDB ID: 6LU7, Chain $A, 2.16 \AA)^{25}$ from protein data bank (PDB) website (www.rcsb.org $)^{26}$. Since there are not yet any solved crystal structures of SARS-CoV-2 PLpro and RdRp proteins, we used the available models. In this regards, we downloaded the high quality model of SARS-CoV-2 PLpro built based on the SARSCoV-2 genome and SARS-CoV PLpro (PDB ID: 5TL6, $2.6 \AA)^{27}$ crystal structure with GMQE and QMEAN scores of 0.92 and -0.22 , respectively ${ }^{28}$. For RdRp, we used the predicted model of SARSCoV-2 RdRp (QHD43415.11.pdb), generated by C-I-TASSER pipeline ${ }^{29}$, which is available from Zhang group's website ${ }^{30}$.

\section{Receptor preparation and defining binding sites}

Prior to the docking analysis, we prepared the three SARS-CoV-2 proteins (Mpro, PLpro, and $\mathrm{RdRp}$ ) by removing water molecules and adding polar hydrogens and partial atomic charges. The receptors were saved in PDBQT format. In order to define the binding sites of the receptors, we retrieved the template ligand structure of the known inhibitors for each of the receptors and identified the ligand-receptor interactions. For the predicted models, we used the inhibitor of the crystal structures that were used for homology modeling. The ligands of the crystal structures fit well in the binding pocket of the modeled structures. We visualized the binding sites for each of the receptors using PyMOL (https://pymol.org/) and SwissPDBViewer ${ }^{31}$ and confirmed our virtualization results with the literature review of the molecular docking studies on SARS and SARS-CoV-2 proteins. Figure 1 illustrates the cartoon structure of Mpro, PLpro, and RdRp with their binding pocket colored in blue color. We defined

grid box size using AutoDock tools (ADT, v1.5.6) ${ }^{32}$ for our docking experiments. Detailed information of the grid box size for each of the receptors is shown in Table 1. We re-docked the known inhibitors of each protein with our grid box information to confirm the chosen grid box information. 


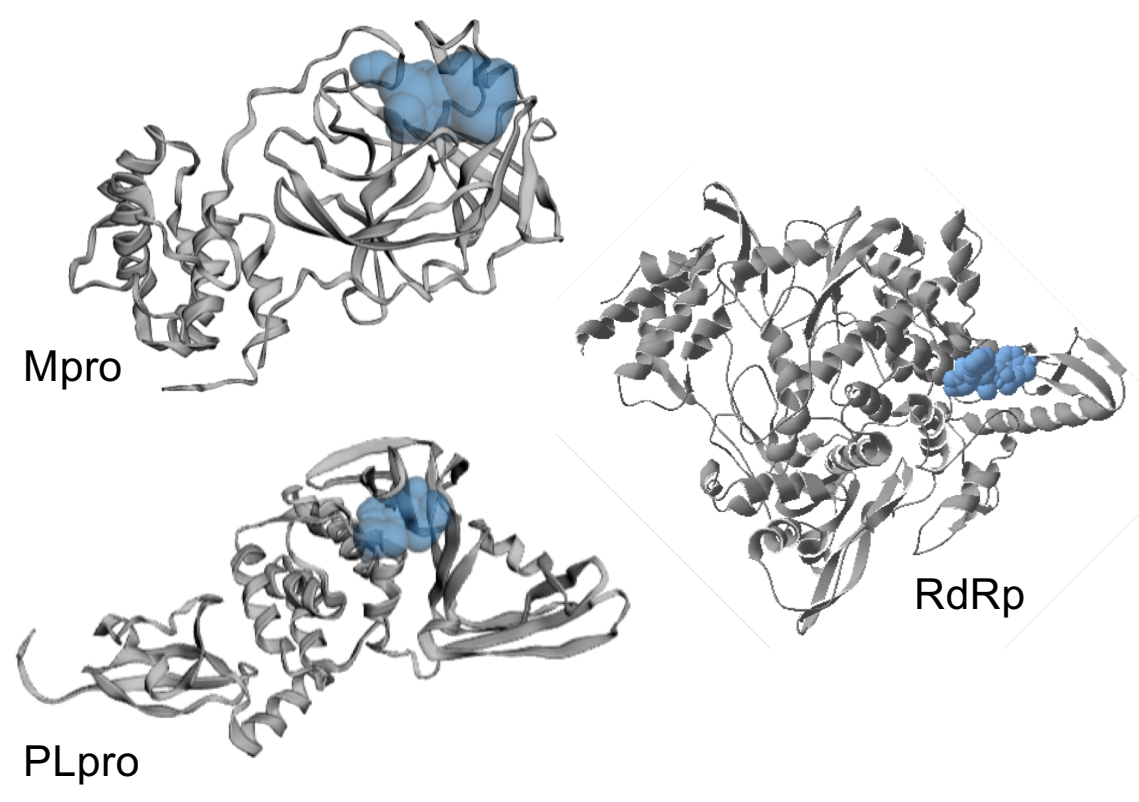

Figure 1. Cartoon representation of SARS-CoV-2 Mpro, PLpro, and RdRp protein structures with their pocket binding sites.

Receptors are represented in gray ribbons. Pocket sites are shown in light blue bobbles.

Table 1. Defined grid box information for three SARS-CoV-2 Mpro, PLpro, and RdRp proteins.

\begin{tabular}{|c|c|c|}
\hline Protein & $\begin{array}{l}\text { Grid box size }(\AA) \\
\text { Centers }(x, y, z)\end{array}$ & Interacting residues \\
\hline Mpro & $\begin{array}{l}50,50,50 \\
-10.850,15.320,68.390\end{array}$ & $\begin{array}{l}\text { Thr24, Thr25, Thr26, His41, Phe140, Leu141, } \\
\text { Asn142, Gly143, Ser144, Cys145, His163, } \\
\text { His164, GLU166, GLN189 }\end{array}$ \\
\hline PLpro & $\begin{array}{l}40,50,40 \\
-7.615,-6.98,-35.36\end{array}$ & $\begin{array}{l}\text { Gly166, Asp167, Pro250, Pro251, Tyr267, } \\
\text { Asn270, Tyr271, Tyr276, Thr304, Asp305 }\end{array}$ \\
\hline RdRp & $\begin{array}{l}40,40,40 \\
6.441,57.178,-16.357\end{array}$ & $\begin{array}{l}\text { Asp221, Asp126, Val128, His133, Leu207, } \\
\text { Asp208, Leu708, Tyr728, Arg733 }\end{array}$ \\
\hline
\end{tabular}

\section{Binding of 1615 FDA approved drugs to SARS-CoV-2 Mpro, PLpro, and RdRp}

We downloaded 1615 available FDA-approved drugs from ZINC15 database ${ }^{33}$ in SDF format and prepared them for docking analysis (see methods section). Once the three SARS-CoV-2 Mpro, PLpro, and RdRp proteins and the 1615 drugs were prepared, we conducted docking analysis using AutoDock Vina ${ }^{34}$ to predict the interaction of drugs with each of the three proteins. We set the AutoDock 
Vina to generate ten poses for each of the ligands to be docked to the protein binding site. AutoDock Vina sorts these ten poses based on their binding energy from lowest to highest. We set all the other parameters as default and used the grid box size information in Table 1 for our docking analysis. Once docking was completed, we chose the best pose of each ligand with the lowest binding energy and the best fit for the receptor pocket site. The result of our docking analysis is reported in Suppl. Table 1, a sorted list of 1600 drugs based on their binding energy with Mpro, PLpro, and RdRp proteins. In order to study the high potential drugs for COVID-19, we decided to look at the top 20 drugs in the list of each receptor (Table 2), excluding those with possible side effects of upper respiratory tract infection, shortness of breath, or throat irritation. These drugs are highlighted in red color in Suppl. Table 1.

Table 2 is the list of the top 20 drugs that bind with lowest binding energy to each of Mpro, PLpro, and RdRp, respectively. Drugs with respiratory side effects are excluded from Table 2. The binding energy values for the top 20 hits are in the range -9.9 to $-8.7,-9.1$ to -7.7 , and -10.7 to $-9.9 \mathrm{kcal} / \mathrm{mol}$ for Mpro, PLpro, and RdRp, respectively. Interestingly, four drugs including Simeprevir, Ledipasvir, Eltrombopag, and Drospirenone appear on the list of all three proteins, see Table 2. Simeprevir is an oral anti hepatitis $\mathrm{C}$ virus (HCV) that blocks the HCV proteases. Ledispasvir is also an antiviral drug and with combination of Sofosbuvir, has been used for $\mathrm{HCV}$ treatment ${ }^{35}$. In consistent with recent virtual screening study, Simeprevir and Ledipasvir have been suggested as potential inhibitors of SARS-CoV$2 \mathrm{Mpro}^{36}$. As for the other two drugs, Drospirenone is a contraceptive containing estrogen and progesterone, and Eltrombopag is to treat thrombocytopenia, both of which might not be suitable for treating coronavirus due to the side effects.

In order to confirm the binding energy values of the docked structures and identify the interacting residues of the proteins with the drugs, we further investigated the ligand-receptor interactions in their docked form using PLIP web server ${ }^{37}$. Figures 2-5 illustrate the potential drugs with lower side effects for COVID-19 and show the interaction of these drugs with Mpro, PLpro, and RdRp binding site residues, respectively. If multiple conformers of a drug are present in the list, only the conformer with lower binding energy is shown. The drugs are shown in orange sticks and the receptors in light blue sticks. Hydrogen bonds (H-bond) and hydrophobic interactions are shown in dark blue and gray color lines, respectively. If there is any salt bridge interaction, it is shown in the yellow sphere. Salt bridges increase the stabilization of the ligand-receptor complex.

\section{Potential SARS-CoV-2 main protein (Mpro) inhibitors}

Based on our docking analysis, potential candidates that inhibit SARS-CoV-2 Mpro protein are listed in the first column of Table $\mathbf{2}$ and the interaction of docked structures are illustrated in Figure 2. 
Idarubicin, the drug in our top list of Mpro inhibitors, binds with the lowest binding energy to this protein and has been suggested as a potential inhibitor for $\mathrm{Mpro}^{38}$. Idarubicin forms six hydrogen bonds with Mpro residues of Thr26, Gly143, Cys145, His163, His164, and Gln189 and further five hydrophobic interactions, making it a promising Mpro inhibitor candidate. Retapamulin (conformer1, ZINC000003931840) and Rifaximin are the two antibiotic drugs in the Mpro list, both of which form one salt bridge interaction with Mpro His41, lowering their binding energy with the protein. Retapamulin forms a hydrogen bond with Arg188 and five hydrophobic interactions. Rifaximin forms five hydrogen bonds of which three are with the same residue, Gln189, and the other two with His41 and Glu166. Both these antibiotics are newly identified SARS-CoV-2 Mpro inhibitors with low binding energy.

Simeprevir, Saquinavir, and Ledipasivir are three antiviral drugs that have been used for $\mathrm{HCV} / \mathrm{HIV}$ treatment, and have been mentioned in recent studies as potential inhibitors of SARS-CoV$2 \mathrm{Mpro}^{36,39}$. Interactions of all these three drugs with the Mpro binding pocket is shown in Figure 2. In summary, Simepivir forms only two H-bonds with Leu141 and Ser144 residues of Mpro and a hydrophobic interaction with Glu166. However, due to a salt bridge interaction with His163, its binding energy is lower than two other antivirals in our list of potential Mpro inhibitors. Saquinavir (conformer1, ZINC000026985532) forms six hydrophobic interactions and six H-bonds with five residues of Phe140, Asn142, Gly143, His163, and Glu166 (2 H-bonds). Ledipasvir, an antiviral at the end of our list, forms three H-bonds with Ser46, Gly143, and Gln189 residues of Mpro and three hydrophobic interactions.

Fosaprepitant and Dolasetron are Antiemetric drugs with minor side effects and are used for aprepitant and nausea treatment, respectively. Fosaprepitant forms two H-bonds with Thr190 (two times) and GIn192. It also forms a salt bridge with His41 and Halogen bonds with Thr25, Ser46, and GIn189, colored in light green in Figure 2. Dolasetron forms a total of five H-bonds with Gly143, Ser144, Cys145, Glu166, and GIn189 and three hydrophobic interactions. Bromocriptine, Ponatinibm Idelalisib, Virapaxar, Eltrombopag, are in the list of potential Mpro inhibitors and have high-risk side effects. A less common side effect of Alprazolam is difficulty of breathing, which makes it less reliable choice of drug for coronavirus treatment. 


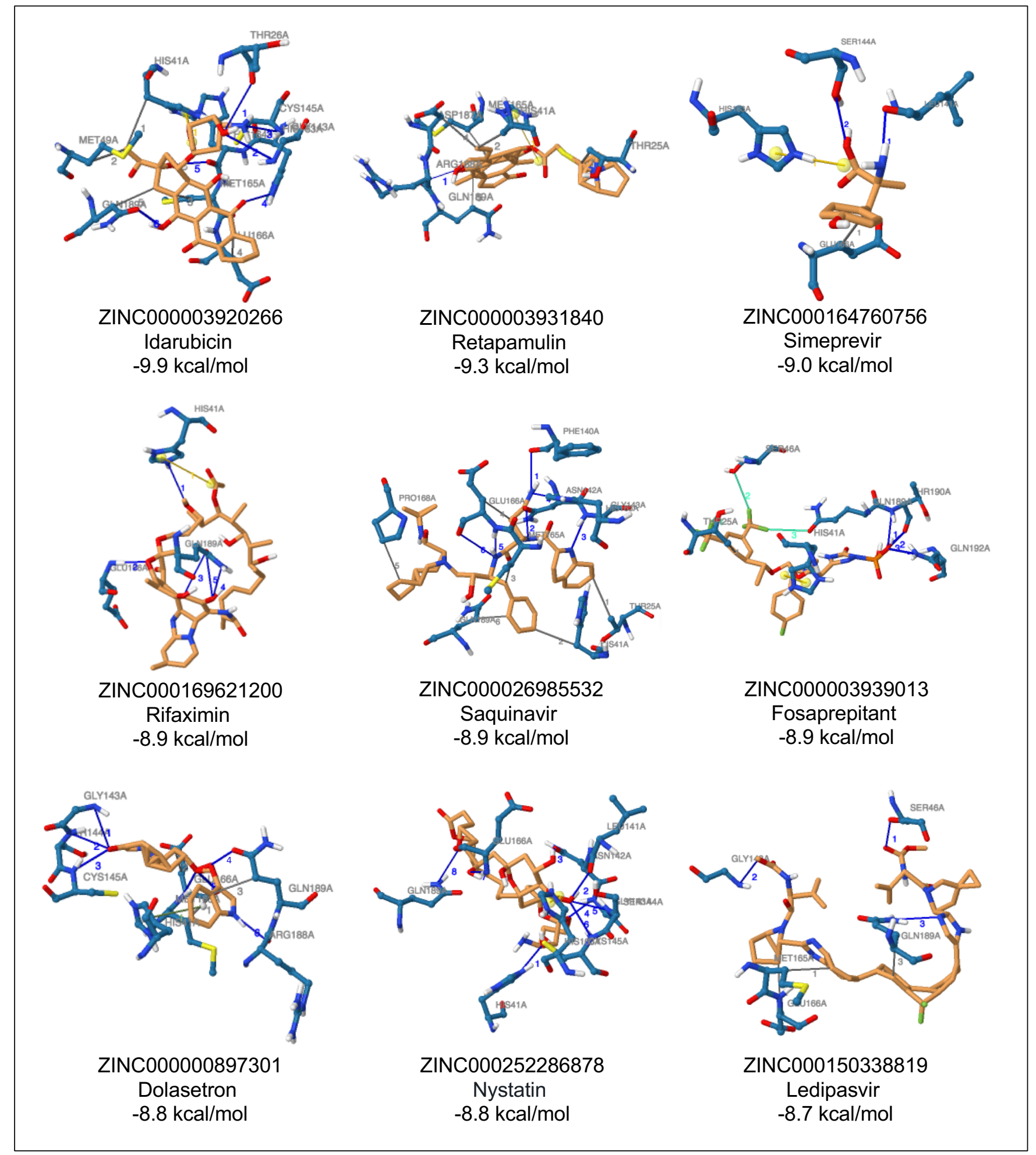

Figure 2. Interaction of the potential drugs with COVID-19 Mpro protein. Ligands are shown in orange sticks and the receptor residues in light blue sticks. $\mathrm{H}$-bonds and hydrophobic interactions are shown with dark blue and gray line, respectively. Salt bridges are shown with yellow spheres. 


\section{Potential SARS-CoV-2 papain-Like protein (PLpro) inhibitors}

Potential inhibitors of SARS-CoV-2 PLpro, resulted from our docking analysis, are listed in the second column of Table 2. Partitaprevir, Glecaprevir, Velpatasvir, Raltegravir, Ledipasvir, Simeprevir are six antiviral (HIV/HCV) drugs. All these drugs except Raltegravir, have been mentioned as potential inhibitors of SARS-CoV-2. Simeprevir appears in our list of PLpro inhibitors as well but with higher binding energy of $(-8.4 \mathrm{kcal} / \mathrm{mol})$ compared to Mpro $(-9 \mathrm{kcal} / \mathrm{mol})$. Figure 3 shows the interactions of potential drugs with PLpro binding residues. Raltegravir forms two H-bonds with Lys 160 and Tyr 276 residues of PLpro along with five hydrophobic interactions. Due to its antiviral property and minor side effects, Raltegravir could be a potential choice of PLpro inhibitor.

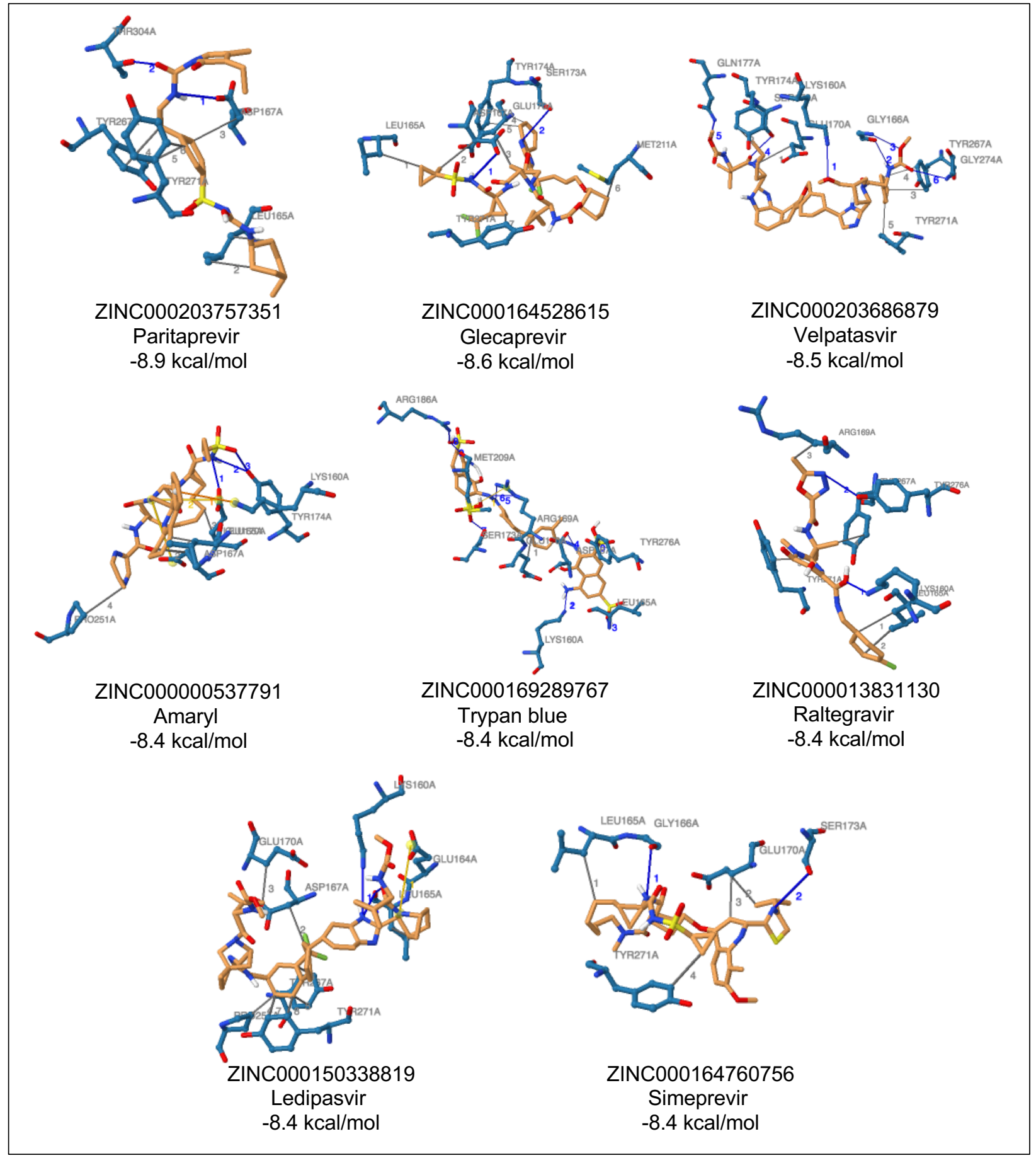


Figure 3. Interaction of the potential drugs with COVID-19 PLpro protein. Ligands are shown in orange sticks and the receptor residues in light blue sticks. H-bonds and hydrophobic interactions are shown with dark blue and gray lines, respectively. Salt bridges are shown with yellow spheres.

Amaryl is an antidiabetic that is used for treatment of type-2 diabetes. Two H-bond interactions of Amaryl with Asp167 and Thr304 residues and six hydrophobic interactions that form the PLpro binding site, resulted in a ligand-receptor complex with low binding energy of $-8.4 \mathrm{kcal} / \mathrm{mol}$. Trypan blue is a dye used in ophthalmic surgeries and it might not be a potential inhibitor. However, studying the scaffold structure of the Trypan blue might help identify other potential inhibitors. Some of the drugs have either serious side effects (Conivaptan, Nilotinib, Spironolactone, Glimepiride, Paliperidone, and Eltrombopag) or interact with antivirals (Nilotinib, Drospirenone, Inspra, and Sonidegib). Digoxin interacts with many drugs and might not be safe to use. Oxazepam may cause respiratory depression if used in high dosages.

\section{Potential SARS-CoV-2 RNA-dependent RNA polymerase (RdRp) inhibitors}

Potential inhibitors of RdRp are listed in the third column of Table 2. Going through the list, some of the drugs have severe side effects or interact with antivirals. There are four antiviral drugs (Paritaprevir, Simeprevir, Glecaprevir, Ledipasvir) in our list as potential RdRp inhibitors and they all have been suggested as possible treatments of COVID-19. Interestingly, three conformers of Simeprevir including ZINC000164760874, ZINC000253632968, and ZINC000164760756 appeared in our list with binding energies of $-10.5,-10$, and $-9.9 \mathrm{kcal} / \mathrm{mol}$, respectively. Three of the antibiotics in our list were Rifabutin, Rifapentine, and Rifaximin. Rifaximin-RdRp complex had $-9.8 \mathrm{kcal} / \mathrm{mol}$ binding energy whereas Rifaximin-Mpro had binding energy of $-8.9 \mathrm{kcal} / \mathrm{mol}$, see Table 2 .

Spinosad is an antiparasite and has been shown to be a potent inhibitor of canine Pglycoprotein ${ }^{40}$. Netupitant is an antiemetic with minor side effects and might be a promising inhibitor of RdRp. Valstar, Eltrombopag, Conivaptan, Nilotinib may have serious side effects. Drospirenone and Sonidegib might interact with HIV treatment medicine. Ecamsule is a chemical used in sunscreens and is not very promising as $\mathrm{RdRp}$ inhibitor. 


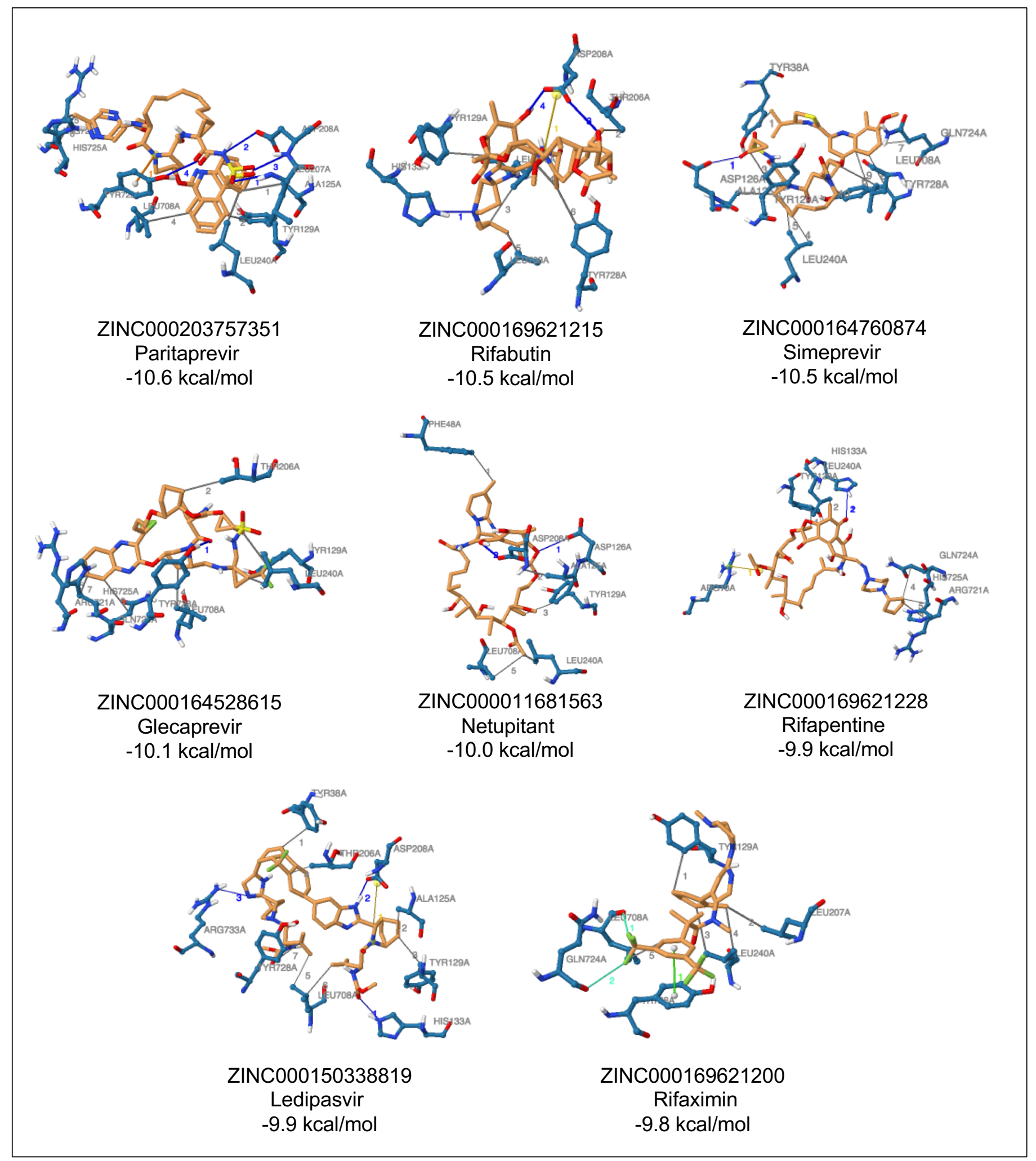

Figure 4. Interaction of the potential drugs with COVID-19 RdRp protein. Ligands are shown in orange sticks and the receptor residues in light blue sticks. H-bonds and hydrophobic interactions are shown with dark blue and gray lines, respectively. Salt bridges are shown with yellow spheres. 
Comparison with current COVID-19 drugs in clinical trials

In order to compare our results to the recent suggested SARS-CoV-2 drugs, we downloaded the structures of Lopinavir, Ritonavir, chloroquine, hydroxychloroquine, and Remdesivir; and we conducted docking analysis for these drugs using our prepared SARS-CoV-2 Mpro, PLpro, and RdRp proteins. Binding energy of the docked complexes is shown in Table $\mathbf{3}$ and their interactions are illustrated in Figure 5.

Table 3. Results of docking analysis of promising SARS-CoV-2 drugs.

\begin{tabular}{cccccc}
\hline \multicolumn{5}{c}{ Binding energy (kcal/mol) } \\
& Remdesivir & Lopinavir & Ritonavir & Chloroquine & Hydroxychloroquine \\
\hline Mpro & -7.9 & -9.3 & -7.6 & -5.8 & -5.8 \\
PLpro & -6.1 & -8.6 & -8.4 & -6.0 & -6.1 \\
RdRp & -8.1 & -10.1 & -8.5 & -5.7 & 5.7 \\
\hline
\end{tabular}

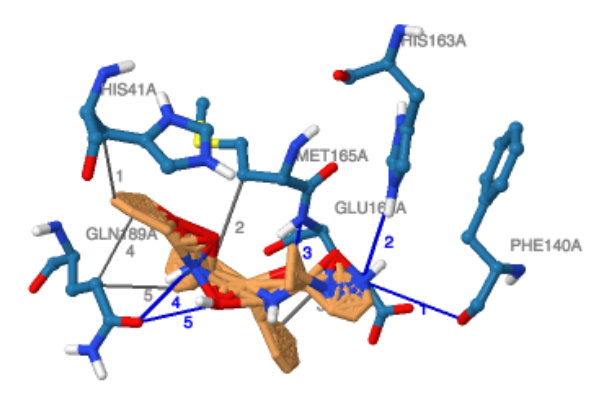

Lopinavir / Mpro

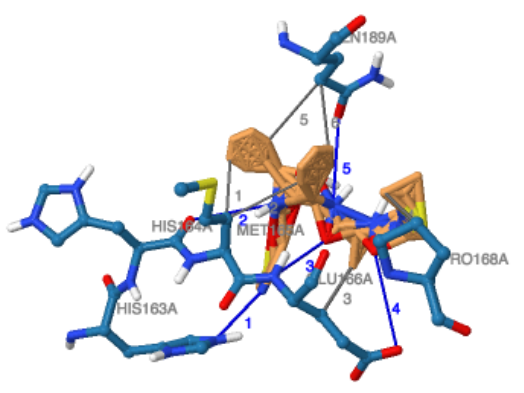

Ritonavir / Mpro

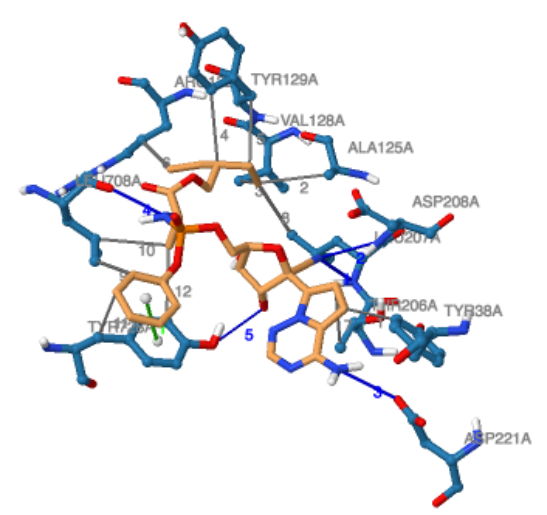

Remdesivir / RdRp
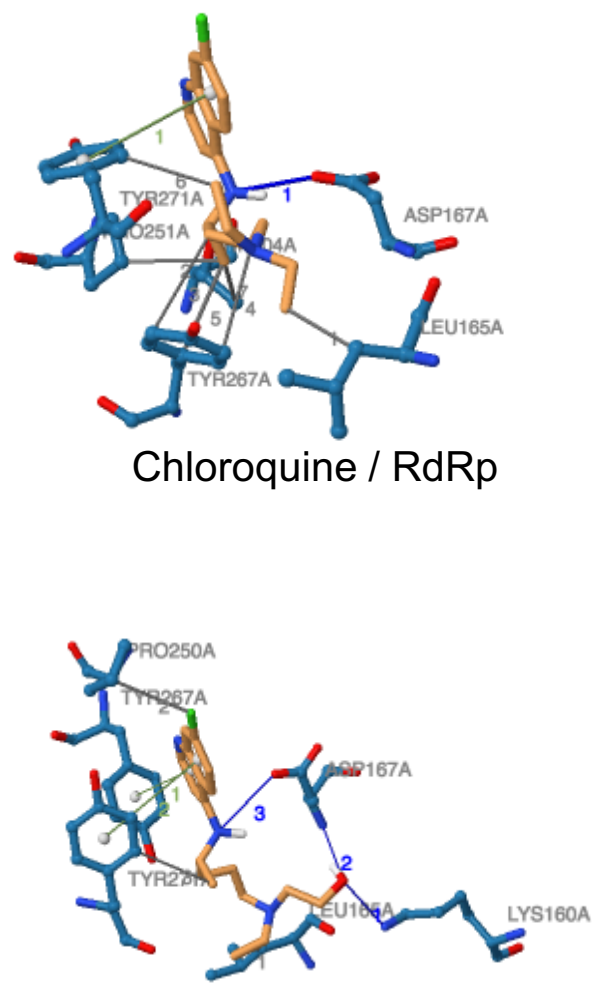

Hydroxychloroquine / RdRp

Figure 5. Ligand-receptor integrations of promising COVID-19 drugs with Mpro, PLpro, and RdRp proteins. These are the structures of the five most promising COVID-19 drugs that are currently on clinical 
trials. The targeted protein was also shown followed by the name of the drug. Ligands are shown in orange sticks and the receptor residues in light blue sticks. $\mathrm{H}$-bonds and hydrophobic interactions are shown with dark blue and gray lines, respectively. Salt bridges are shown with yellow spheres.

Remdesevir, a potential inhibitor of RdRp, had a low binding energy of $-8.1 \mathrm{kcal} / \mathrm{mol}$, which may be a promising inhibitor or SARS-CoV-2. Lopinavir and Ritanovir were suggested inhibitors of Mpro. Interestingly, our analysis showed a lower binding energy for these drugs with $\operatorname{RdRp}(-10.1$ and -8.5 $\mathrm{kcal} / \mathrm{mol})$ compared to Mpro $(-9.3$ and $-7.6 \mathrm{kcal} / \mathrm{mol})$, suggesting that they are good inhibitors of RdRp as well. Chloroquine and hydroxychloroquine are suggested as potential inhibitors of PLpro ${ }^{22}$, but our docking analysis showed a high binding energy for these ligands, suggesting that the antiviral effects of chloroquine may be mainly at the entry-level instead of post-entry stage.

\section{Discussion}

The pandemic of COVID-19 has become a global emergency. Scientists and physicians are searching for potential drugs for COVID-19 treatment, and there is an urgent need to identify effective drugs, with lower side effects to fight against SARS-CoV-2. With this purpose, we conducted a virtual screening of 1615 FDA-approved drugs targeting three important non-structural proteins of SARS-CoV2, main protease (Mpro), papain-like protease (PLpro), and RNA-dependent RNA polymerase (RdRp) using Autodock Vina. The crystal structure of the SARS-CoV-2 Mpro from Protein Data Bank and models of PLpro and RdRp proteins were used. Our results showed that some of the known HIV/HCV antiviral drugs are promising inhibitors of SARS-CoV-2. Simeprevir and Ledipasvir showed low binding energy with all three targets. Our results confirmed some of the suggested SARS-CoV-2 inhibitors including: Idarubicin Simeprevir, Saquinavir, Ledipasivir, Partitaprevir, Glecaprevir, and Velpatasvir.

Furthermore, we manually studied the drugs with lower binding energy against SARS-CoV-2 Mpro, PLpro, and RdRp proteins and lower side effects. We found seven new FDA-approved drugs with lower binding energy against SARS-CoV-2 targets compared to the current under clinical trial drugs and these seven new drugs can be considered as potential candidate drugs subject to clinical tests. Our suggested candidate drugs are comprised of four antibiotics, two antiemetics, and an antiviral drug with low side effects including: Retapamulin (antibiotic), Rifimixin (antibiotic), and Fosaprepitant (antiemetic) for Mpro; Furthermore, Amaryl (antidiabetic) and Raltegravir (antiviral) for PLpro; Rifabutin (antibiotic), Netupitant (antiemetic), and Rifimixin (antibiotic) for RdRP. Although the computational docking analysis has some limitation and further validation study is needed, our virtual screening based on the low binding energy which suggested some new drugs, e.g., Rifabutin and Raltegravir, respectively, with a binding energy ranging from -10.5 to $-8.4 \mathrm{kcal} / \mathrm{mol}$, will provide some first-hand 
information in selecting the promising drugs. We plotted the interactions of our suggested potential drugs with the SARS-CoV-2 proteins to further help in choosing the optimized drugs.

We also conducted docking analysis on five drugs that have been suggested as promising SARS-CoV-2 inhibitors which are under clinical trials including Remdesivir, Lopinavir, Ritonavir, chloroquine, and hydroxychloroquine. Our docking results showed low binding energy for Remdesivir, Lopinavir, and Ritonavir with all three proteins and confirming them as promising SARS-CoV-2 inhibitors. However, chloroquine, and hydroxychloroquine on the other hand, had high binding energy with all three proteins, suggesting that they may work mainly through other mechanisms (e.g., immunomodulation) in COVID-19 treatment. We hope that our findings can help in finding the best treatments for COVID-19.

\section{Methods}

We performed molecular docking analysis of FDA-approved drug with the binding pocket of three different SARS-CoV-2 NSPs to identify potential drugs for treatment. The newly released crystal structure of SARS-CoV-2 (PDB ID: 6LU7, Chain A, $2.16 \AA)^{25}$ was retrieved from protein data bank (PDB) website (www.rcsb.org) ${ }^{26}$. High quality model of SARS-CoV-2 PLpro built based on the SARSCoV-2 genome and SARS-CoV PLpro (PDB ID: 5TL6, $2.6 \AA)^{27}$ crystal structure with GMQE and QMEAN scores of 0.92 and -0.22 , respectively, were downloaded to be used as PLpro receptor ${ }^{28}$. Predicted model of SARS-CoV-2 RdRp (QHD43415.11.pdb), generated by C-I-TASSER pipeline ${ }^{29}$, available from Zhang group's website (https://zhanglab.ccmb.med.umich.edu/C-I-TASSER/2019nCov/, were used for RdRp receptor.

Three receptor molecules and 1615 Ligands were prepared using AutoDockTools (ADT, v1.5.6) to be used as input files for the docking analysis. For preparation of protein input files, all water molecules, ligands and ions were removed and polar hydrogens were added from pdb file ./prepare_receptor4.py command of the Autodocktools. Kollman-united charge was used to calculate the partial atomic charge and the prepared file was saved in format to be used in the following steps. Three-dimension (3D) structures of FDA-approved drugs, containing 1615 compounds, were downloaded from ZINC15 database ${ }^{33}$ in structure-data file (SDF) format. Then OpenBabel (v2.3.1) ${ }^{41}$ was used to separate the files, add hydrogen bonds and assign Rotatable bonds and Gasteiger-Marsili charges. Finally, all the ligands were saved in PDBQT for further docking processes. AutoDockTools 4.2 was used to identify grid box size and center, see Table 1 for detailed information. Before starting the docking analysis of FDA-approved drugs. The co-crystal ligand of each structure was re-docked to as an inhibitor to ensure the valid box rigid information. Docking processes were performed using Autodock Vina ${ }^{34}$ with the default parameters on a Linux system connected to a server with $144 \mathrm{CPU}$. 
For each ligand, ten poses were generated and docked into the protein binding site. All these poses were sorted according to their binding affinities. Docking processes were done using written in-house scripts. All visualizations were done using SwissPDBViewer ${ }^{31}$, PyMOL (https://pymol.org/) and PLIP webserver ${ }^{37}$.

\section{Conflicts of interests}

The authors declare no conflict of interest.

\section{References}

1. Wu, F. et al. A new coronavirus associated with human respiratory disease in China. Nature 579, 265-269 (2020).

2. Huang, C. et al. Clinical features of patients infected with 2019 novel coronavirus in Wuhan, China. The Lancet 395, 497-506 (2020).

3. Ruan, S. Likelihood of survival of coronavirus disease 2019. The Lancet Infectious Diseases (2020).

4. WHO Update 49-SARS case fatality ratio, incubation period. https://www.who.int/csr/sars/archive/2003 05 07a/en/. (May 7, 2003).

5. WHO Middle East respiratory syndrome coronavirus (MERS-CoV): key facts. https://www.who.int/en/news-room/fact-sheets/detail/middle-east-respiratory-syndromecoronavirus-(mers-cov). (March 11, 2019).

6. Rothe, C. et al. Transmission of 2019-nCoV Infection from an Asymptomatic Contact in Germany. N Engl J Med 382, 970-971 (2020).

7. Verity, R. et al. Estimates of the severity of coronavirus disease 2019: a model-based analysis. The Lancet Infectious Diseases (2020).

8. Wu, C. et al. Analysis of therapeutic targets for SARS-CoV-2 and discovery of potential drugs by computational methods. Acta Pharmaceutica Sinica B (2020).

9. Guo, Y.R. et al. The origin, transmission and clinical therapies on coronavirus disease 2019 (COVID-19) outbreak - an update on the status. Mil Med Res 7, 11 (2020).

10. Ziebuhr, J. \& Siddell, S.G. Processing of the human coronavirus 229E replicase polyproteins by the virus-encoded $3 \mathrm{C}$-like proteinase: identification of proteolytic products and cleavage sites common to pp1a and pp1ab. J Virol 73, 177-185 (1999).

11. Barretto, N. et al. The papain-like protease of severe acute respiratory syndrome coronavirus has deubiquitinating activity. Journal of virology 79, 15189-15198 (2005).

12. Snijder, E.J. et al. Unique and conserved features of genome and proteome of SARScoronavirus, an early split-off from the coronavirus group 2 lineage. Journal of molecular biology 331, 991-1004 (2003).

13. Baden, L.R. \& Rubin, E.J. Covid-19 - The Search for Effective Therapy. N Engl J Med (2020).

14. Monteleone, G., Sarzi-Puttini, P.C. \& Ardizzone, S. Preventing COVID-19-induced pneumonia with anticytokine therapy. The Lancet Rheumatology (2020).

15. Matthay, M.A., Aldrich, J.M. \& Gotts, J.E. Treatment for severe acute respiratory distress syndrome from COVID-19. The Lancet Respiratory Medicine (2020).

16. Wang, M. et al. Remdesivir and chloroquine effectively inhibit the recently emerged novel coronavirus (2019-nCoV) in vitro. Cell Res 30, 269-271 (2020).

17. Chu, C.M. et al. Role of lopinavir/ritonavir in the treatment of SARS: initial virological and clinical findings. Thorax 59, 252-256 (2004). 
18. Chan, J.F. et al. Treatment With Lopinavir/Ritonavir or Interferon-beta1b Improves Outcome of MERS-CoV Infection in a Nonhuman Primate Model of Common Marmoset. J Infect Dis 212, 1904-1913 (2015).

19. Kim, U.J., Won, E.J., Kee, S.J., Jung, S.I. \& Jang, H.C. Combination therapy with lopinavir/ritonavir, ribavirin and interferon-alpha for Middle East respiratory syndrome. Antivir Ther 21, 455-459 (2016).

20. Cao, B. et al. A Trial of Lopinavir-Ritonavir in Adults Hospitalized with Severe Covid-19. N Engl $J$ Med (2020).

21. Gao, J., Tian, Z. \& Yang, X. Breakthrough: Chloroquine phosphate has shown apparent efficacy in treatment of COVID-19 associated pneumonia in clinical studies. Bioscience trends (2020).

22. Rimanshee, A., Amit, D., Vishal, P. \& Mukesh, K. (ChemRxiv. Preprint.; 2020).

23. Xu, Z. et al. Nelfinavir was predicted to be a potential inhibitor of 2019-nCov main protease by an integrative approach combining homology modelling, molecular docking and binding free energy calculation. bioRxiv, 2020.2001.2027.921627 (2020).

24. Liu, X. \& Wang, X.-J. Potential inhibitors for 2019-nCoV coronavirus M protease from clinically approved medicines. bioRxiv, 2020.2001.2029.924100 (2020).

25. Liu, X., Zhang, B., Jin, Z., Yang, H. \& Rao, Z. (To be published. ; 2020).

26. H.M, B. et al. ( Nucleic Acids Research , 28: 235-242; 2000).

27. Daczkowski, C.M. et al. Structural Insights into the Interaction of Coronavirus Papain-Like Proteases and Interferon-Stimulated Gene Product 15 from Different Species. J Mol Biol 429, 1661-1683 (2017).

28. Martin, S. Homology Models of the Papain-Like Protease PLpro from Coronavirus 2019-nCoV. (2020).

29. Zheng, W. et al. Deep-learning contact-map guided protein structure prediction in CASP13. Proteins 87, 1149-1164 (2019).

30. Chengxin, Z. et al. (Journal of Proteome Research; 2020).

31. Guex, N. \& Peitsch, M.C. SWISS-MODEL and the Swiss-PdbViewer: an environment for comparative protein modeling. Electrophoresis 18, 2714-2723 (1997).

32. Morris, G.M. et al. AutoDock4 and AutoDockTools4: Automated docking with selective receptor flexibility. J Comput Chem 30, 2785-2791 (2009).

33. Sterling, T. \& Irwin, J.J. ZINC 15--Ligand Discovery for Everyone. J Chem Inf Model 55, 23242337 (2015).

34. Trott, O. \& Olson, A.J. AutoDock Vina: improving the speed and accuracy of docking with a new scoring function, efficient optimization, and multithreading. J Comput Chem 31, 455-461 (2010).

35. Kwok, R.M. et al. Sofosbuvir plus ledispasvir for recurrent hepatitis $C$ in liver transplant recipients. Liver Transpl 22, 1536-1543 (2016).

36. Chen, Y.W., Yiu, C.-P. \& Wong, K.-Y. (ChemRxiv. Preprint. ; 2020).

37. Salentin, S., Schreiber, S., Haupt, V.J., Adasme, M.F. \& Schroeder, M. PLIP: fully automated protein-ligand interaction profiler. Nucleic Acids Research 43, W443-W447 (2015).

38. Rani, R., Singh, A., Pareek, A. \& Tomar, S. (ChemRxiv; 2020).

39. Dayer, M.R., Taleb-Gassabi, S. \& Dayer, M.S. Lopinavir; A Potent Drug against Coronavirus Infection: Insight from Molecular Docking Study. Archives of Clinical Infectious Diseases 12 (2017).

40. Schrickx, J.A. Spinosad is a potent inhibitor of canine P-glycoprotein. Vet J 200, 195-196 (2014).

41. O'Boyle, N.M. et al. Open Babel: An open chemical toolbox. J Cheminform 3, 33 (2011). 